TRANSACTIONS OF THE

AMERICAN MATHEMATICAL SOCIETY

Volume 361, Number 6, June 2009, Pages 3031-3040

S 0002-9947(08)04563-7

Article electronically published on December 29, 2008

\title{
TAUBERIAN CONDITIONS FOR GEOMETRIC MAXIMAL OPERATORS
}

\author{
PAUL HAGELSTEIN AND ALEXANDER STOKOLOS
}

\begin{abstract}
Let $\mathcal{B}$ be a collection of measurable sets in $\mathbb{R}^{n}$. The associated geometric maximal operator $M_{\mathcal{B}}$ is defined on $L^{1}\left(\mathbb{R}^{n}\right)$ by $M_{\mathcal{B}} f(x)=$ $\sup _{x \in R \in \mathcal{B}} \frac{1}{|R|} \int_{R}|f|$. If $\alpha>0, M_{\mathcal{B}}$ is said to satisfy a Tauberian condition with respect to $\alpha$ if there exists a finite constant $C$ such that for all measurable sets $E \subset \mathbb{R}^{n}$ the inequality $\left|\left\{x: M_{\mathcal{B} \chi_{E}}(x)>\alpha\right\}\right| \leq C|E|$ holds. It is shown that if $\mathcal{B}$ is a homothecy invariant collection of convex sets in $\mathbb{R}^{n}$ and the associated maximal operator $M_{\mathcal{B}}$ satisfies a Tauberian condition with respect to some $0<\alpha<1$, then $M_{\mathcal{B}}$ must satisfy a Tauberian condition with respect to $\gamma$ for all $\gamma>0$ and moreover $M_{\mathcal{B}}$ is bounded on $L^{p}\left(\mathbb{R}^{n}\right)$ for sufficiently large $p$. As a corollary of these results it is shown that any density basis that is a homothecy invariant collection of convex sets in $\mathbb{R}^{n}$ must differentiate $L^{p}\left(\mathbb{R}^{n}\right)$ for sufficiently large $p$.
\end{abstract}

Let $\mathcal{B}$ be a collection of measurable sets in $\mathbb{R}^{n}$. We define the associated geometric maximal operator $M_{\mathcal{B}}$ on $L^{1}\left(\mathbb{R}^{n}\right)$ by

$$
M_{\mathcal{B}} f(x)=\sup _{x \in R \in \mathcal{B}} \frac{1}{|R|} \int_{R}|f| .
$$

The operator $M_{\mathcal{B}}$ is said to satisfy a Tauberian condition with respect to $\alpha$ if there exists a finite constant $C$ such that for any measurable set $E \subset \mathbb{R}^{n}$ the inequality

$$
\left|\left\{x: M_{\mathcal{B}} \chi_{E}(x)>\alpha\right\}\right| \leq C|E|
$$

holds.

This is a very weak condition on a maximal operator - weaker in fact than a restricted weak type $(1,1)$ estimate. This is a useful condition on a maximal operator, however, as was shown by A. Córdoba and R. Fefferman in their work relating the $L^{p}$ bounds of certain multiplier operators to the weak type $\left(\left(\frac{p}{2}\right)^{\prime},\left(\frac{p}{2}\right)^{\prime}\right)$ bounds of associated geometric maximal operators. (See [2] for complete details.)

Now, suppose we are given a maximal operator $M_{\mathcal{B}}$ satisfying a Tauberian condition such as, for instance,

$$
\left|\left\{x: M_{\mathcal{B} \chi_{E}}(x)>\frac{3}{4}\right\}\right| \leq C|E| .
$$

Received by the editors September 12, 2006 and, in revised form, May 30, 2007.

2000 Mathematics Subject Classification. Primary 42B25.

The first author's research was partially supported by the Baylor University Summer Sabbatical Program.

The second author's research was partially supported by the DePaul University Research Council Leave Program.

(C)2008 American Mathematical Society Reverts to public domain 28 years from publication 
One might wonder whether or not $M_{\mathcal{B}}$ must be bounded on $L^{p}\left(\mathbb{R}^{n}\right)$ for $p>1$ or whether or not $M_{\mathcal{B}}$ must satisfy any given stronger Tauberian estimate, say, $\left|\left\{x: M_{\mathcal{B}} \chi_{E}(x)>\frac{1}{4}\right\}\right| \leq C^{\prime}|E|$. That neither of the above holds, even in the case that $\mathcal{B}$ is a homothecy invariant collection of sets, can be seen by the following example. (Recall that a collection of sets in $\mathbb{R}^{n}$ is said to be homothecy invariant if and only if any translate or dilate of any member of the collection also lies in the collection.)

Example. Let $\mathcal{B}$ be the collection of sets in $\mathbb{R}^{1}$ of the form $I_{1} \cup I_{2}$, where $I_{1}$ and $I_{2}$ are intervals and $\left|I_{2}\right|=2\left|I_{1}\right|$. Note $\mathcal{B}$ is homothecy invariant. $M_{\mathcal{B}}$ is not bounded on $L^{p}\left(\mathbb{R}^{1}\right)$ for $1 \leq p<\infty$, as $M_{\mathcal{B}} \chi_{[0,1]}(x) \geq \frac{1}{3}$ for all $x$ in $\mathbb{R}^{1}$. Moreover $\left|\left\{x: M_{\mathcal{B}} \chi_{[0,1]}(x)>\frac{1}{4}\right\}\right|=\infty$, and so $M_{\mathcal{B}}$ does not satisfy a Tauberian condition with respect to $\frac{1}{4}$.

$M_{\mathcal{B}}$ does satisfy a Tauberian condition with respect to $\frac{3}{4}$, however. To see this, let $E$ be a set of finite measure, and let $\left\{A_{j}\right\} \subset \mathcal{B}$ be such that $\frac{1}{\left|A_{j}\right|} \int_{A_{j}} \chi_{E}>\frac{3}{4}$ for each $j$. Now, each $A_{j}$ is of the form $A_{j}=A_{j}^{1} \cup A_{j}^{2}$, where $A_{j}^{1}$ and $A_{j}^{2}$ are intervals and $2\left|A_{j}^{1}\right|=\left|A_{j}^{2}\right|$. Since $\frac{1}{\left|A_{j}\right|} \int_{A_{j}} \chi_{E}>\frac{3}{4}$, we must have $\frac{1}{\left|A_{j}^{1}\right|} \int_{A_{j}^{1}} \chi_{E}>\frac{1}{4}$ and $\frac{1}{\left|A_{j}^{2}\right|} \int_{A_{j}^{2}} \chi_{E}>\frac{1}{4}$. So by the Vitali Covering Theorem we must have $\left|\cup A_{j}^{1}\right| \leq 12|E|$ and $\left|\cup A_{j}^{2}\right| \leq 12|E|$. Therefore $\left|\cup A_{j}\right| \leq 24|E|$ and hence $\left|\left\{x: M_{\mathcal{B}} \chi_{E}(x)>\frac{3}{4}\right\}\right| \leq 24|E|$.

Note that in the above example the elements of $\mathcal{B}$ are not all convex. The primary purpose of this paper is to show that if $\mathcal{B}$ is a homothecy invariant collection of convex sets in $\mathbb{R}^{n}$ and the associated maximal operator $M_{\mathcal{B}}$ satisfies a Tauberian condition with respect to some $0<\alpha<1$, then $M_{\mathcal{B}}$ must satisfy a Tauberian condition with respect to $\gamma$ for every $\gamma>0$. As a corollary of the proof we shall see that if $\mathcal{B}$ is a homothecy invariant collection of convex sets and $M_{\mathcal{B}}$ satisfies a Tauberian condition with respect to $\alpha$ for some $0<\alpha<1$, then $M_{\mathcal{B}}$ must be bounded on $L^{p}\left(\mathbb{R}^{n}\right)$ for sufficiently large $p$. As a further corollary we shall see that any density basis that is a homothecy invariant collection of convex sets in $\mathbb{R}^{n}$ must differentiate $L^{p}\left(\mathbb{R}^{n}\right)$ for sufficiently large $p$.

Our proof will consist of two main parts. First we shall show the desired result in the special case that $\mathcal{B}$ is a homothecy invariant collection of rectangular parallelepipeds. Secondly we shall reduce the general case involving homothecy invariant collections of convex sets to this special case.

Proposition 1. Let $\mathcal{B}$ be a homothecy invariant collection of rectangular parallelepipeds in $\mathbb{R}^{n}$. Suppose for some $0<\gamma<1$ there exists $0<C_{\gamma}<\infty$ such that

$$
\left|\left\{x: M_{\mathcal{B}} \chi_{E}(x)>\gamma\right\}\right| \leq C_{\gamma}|E|
$$

holds for all measurable sets $E$ in $\mathbb{R}^{n}$. Then if $\alpha>0$, there exists $0<C_{\alpha, \gamma}<\infty$ such that

$$
\left|\left\{x: M_{\mathcal{B} \chi_{E}}(x)>\alpha\right\}\right| \leq C_{\alpha, \gamma}|E|
$$

holds for all measurable sets $E$ in $\mathbb{R}^{n}$, where $C_{\alpha, \gamma}$ depends only on $C_{\gamma}, \alpha, \gamma$, and the dimension $n$.

Proof. If $\alpha \geq \gamma$, then we may trivially set $C_{\alpha, \gamma}=C_{\gamma}$. So we assume without loss of generality that $0<\alpha<\gamma$. Let $E$ be a measurable set in $\mathbb{R}^{n}$. We inductively 
define $\mathcal{H}_{\mathcal{B}, \gamma}^{k}(E)$ for $k=0,1,2, \ldots$ by setting $\mathcal{H}_{\mathcal{B}, \gamma}^{0}(E)=E$ and

$$
\mathcal{H}_{\mathcal{B}, \gamma}^{k}(E)=\left\{x: M_{\mathcal{B}} \chi_{\mathcal{H}_{\mathcal{B}, \gamma}^{k-1}(E)}(x) \geq \gamma\right\}
$$

for $k \geq 1$.

Lemma 1. Suppose $R \in \mathcal{B}$ and $\frac{1}{|R|} \int_{R} \chi_{E}=\alpha$. Then $R \subset \mathcal{H}_{\mathcal{B}, \gamma}^{K_{\alpha, \gamma}}(E)$ for some constant $K_{\alpha, \gamma}$ depending only on $n$, $\alpha$, and $\gamma$.

Proof. Let $Q$ denote the unit $n$-cube $[0,1]^{n}$ in $\mathbb{R}^{n}$. Now, since $R$ is a rectangular parallelepiped, there exists a linear bijection $\Lambda: \mathbb{R}^{n} \rightarrow \mathbb{R}^{n}$ such that $\{\Lambda(x): x \in$ $R\}=Q$.

For each set $S \in \mathbb{R}^{n}$ let

$$
S_{\Lambda}=\{\Lambda(x): x \in S\}
$$

Also, let

$$
\mathcal{B}_{\Lambda}=\left\{S_{\Lambda}: S \in \mathcal{B}\right\} .
$$

Note if $U$ and $V$ are measurable sets in $\mathbb{R}^{N}$ and $|V| \neq 0$, then $\frac{|U|}{|V|}=\frac{\left|U_{\Lambda}\right|}{\left|V_{\Lambda}\right|}$. Hence $M_{\mathcal{B}} \chi_{E} \geq \alpha$ on a set $S$ in $\mathcal{B}$ if and only if $M_{\mathcal{B}_{\Lambda}} \chi_{E_{\Lambda}} \geq \alpha$ on $S_{\Lambda}$. Now, if $\left\{x: M_{\mathcal{B}} \chi_{E}(x) \geq \alpha\right\}=\cup S_{j}$ it follows that $\left\{x: M_{\mathcal{B}_{\Lambda}} \chi_{E_{\Lambda}}(x) \geq \alpha\right\}=\cup S_{j_{\Lambda}}$. As $\left(\cup S_{j}\right)_{\Lambda}=\cup S_{j_{\Lambda}}$ one then sees that

$$
\left(\mathcal{H}_{\mathcal{B}, \gamma}^{k}(E)\right)_{\Lambda}=\mathcal{H}_{\mathcal{B}_{\Lambda}, \gamma}^{k}\left(E_{\Lambda}\right)
$$

holds for any positive integer $k$. As $R_{\Lambda}=Q$ we realize it suffices to prove

$$
Q \subset \mathcal{H}_{\mathcal{B}_{\Lambda}, \gamma}^{K_{\alpha, \gamma}}\left(E_{\Lambda}\right)
$$

for some constant $K_{\alpha, \gamma}$ depending only on $n, \alpha$, and $\gamma$. As $\int_{Q} \chi_{E_{\Lambda}}>\alpha$ and $Q \in \mathcal{B}_{\Lambda}$ we then realize it suffices to prove the lemma in the special case that $R=Q$. Note that as $\mathcal{B}$ is homothecy invariant we may also assume without loss of generality that any $n$-cube in $\mathbb{R}^{n}$ with sides parallel to the axes lies in $\mathcal{B}$.

So, we now suppose without loss of generality that $R=Q$, all $n$-cubes in $\mathbb{R}^{n}$ whose sides are parallel to the axes lie in $\mathcal{B}$, and $\int_{Q} \chi_{E}=\alpha$. We take the CalderónZygmund decomposition of $\chi_{E \cap Q}$ with respect to $\gamma$ yielding a collection of cubes $\left\{Q_{j}\right\}$ in $Q$ with sides parallel to the axes. In particular the collection of cubes $\left\{Q_{j}\right\}$ is such that $\frac{1}{\left|Q_{j}\right|} \int_{Q_{j}} \chi_{E}>\gamma$ for each $j$ and $E \cap Q \subset \cup Q_{j}$ almost everywhere. Note that none of the cubes $Q_{j}$ is $Q$ itself, as $\frac{1}{|Q|} \int_{Q} \chi_{E}=\alpha<\gamma$. Also note that each $Q_{j}$ is a dyadic cube and hence has a unique parent dyadic cube. For any constant $c>1$, we let $c Q_{j}$ denote the cube containing $Q_{j}$ that has sidelength $c$ times that of $Q_{j}$ and also has a common corner with $Q_{j}$ and the parent cube of $Q_{j}$.

Let now $E_{0}=E \cap Q, E_{1}=\cup Q_{j}$, and, for $k \geq 2$,

$$
E_{k}=\bigcup_{j}\left(\frac{1}{\gamma}\right)^{(k-1) / n} Q_{j} .
$$

Note that since

$$
\frac{\left|\left(\frac{1}{\gamma}\right)^{\frac{k}{n}} Q_{j}\right|}{\left|\left(\frac{1}{\gamma}\right)^{\frac{k+1}{n}} Q_{j}\right|}=\gamma
$$


we have $M_{\mathcal{B}} \chi_{E_{k}} \geq \gamma$ on $E_{k+1}$. Also observe that since the average of $\chi_{E}$ over each $Q_{k}$ exceeds $\gamma$ we have $E_{1} \subset \mathcal{H}_{\mathcal{B}, \gamma}^{1}(E)$, and as $M_{\mathcal{B}} \chi_{E_{k}} \geq \gamma$ on $E_{k+1}$ we have $E_{k} \subset \mathcal{H}_{\mathcal{B}, \gamma}^{k}(E)$ for each $k$.

Now let $N$ be a positive integer such that $\left(\frac{1}{\gamma}\right)^{N} \geq \gamma \cdot 2^{n}$. Let $Q_{j}^{*}$ denote the parent cube of $Q_{j}$. Now, since

$$
\frac{\left|\left(\frac{1}{\gamma}\right)^{\frac{N}{n}} Q_{j}\right|}{\left|Q_{j}^{*}\right|} \geq\left(\frac{1}{\gamma}\right)^{N} \cdot \frac{1}{2^{n}} \geq \gamma
$$

we have

$$
\frac{\left|E_{N+1} \cap Q_{j}^{*}\right|}{\left|Q_{j}^{*}\right|} \geq \gamma
$$

and so $M_{\mathcal{B}} \chi_{E_{N+1}} \geq \gamma$ on $Q_{j}^{*}$.

Now let $Q_{j_{1}}, Q_{j_{2}}, \ldots$ be elements of $\left\{Q_{j}\right\}$ such that the $Q_{j_{i}}^{*}$ have disjoint interiors and such that $\left|\cup Q_{j_{i}}^{*}\right|=\left|\cup Q_{k}^{*}\right|$. Note that each $Q_{j_{i}}^{*}$ is contained in $Q$ since $Q \notin\left\{Q_{i}\right\}$. Note also that $\left|E \cap Q_{j_{k}}^{*}\right| \leq \gamma\left|Q_{j_{k}}^{*}\right|$, as otherwise $Q_{j_{k}}^{*}$ would have been a selected $Q_{j}$. Hence we have

$$
\begin{aligned}
\left|\left\{x \in Q: M_{\mathcal{B}} \chi_{E_{N+1}}(x) \geq \gamma\right\}\right| & \geq\left|\cup Q_{j}^{*}\right| \\
& =\sum\left|Q_{j_{k}}^{*}\right| \\
& \geq \frac{1}{\gamma} \sum\left|E \cap Q_{j_{k}}^{*}\right| \\
& \geq \frac{1}{\gamma}\left|E_{0}\right| .
\end{aligned}
$$

In particular,

$$
\left|Q \cap \mathcal{H}_{\mathcal{B}, \gamma}^{N+2}(E)\right| \geq \frac{1}{\gamma}\left|E_{0}\right| .
$$

Note that if $\left|Q \cap \mathcal{H}_{\mathcal{B}, \gamma}^{N+2}(E)\right| \geq \gamma$ we have $Q \subset \mathcal{H}_{\mathcal{B}, \gamma}^{(N+2)+1}(E)$. Otherwise by the above argument we may obtain

$$
\begin{aligned}
\left|Q \cap \mathcal{H}_{\mathcal{B}, \gamma}^{2(N+2)}(E)\right| & \geq \frac{1}{\gamma}\left|H_{\mathcal{B}, \gamma}^{N+2}(E) \cap Q\right| \\
& \geq\left(\frac{1}{\gamma}\right)^{2}\left|E_{0}\right| .
\end{aligned}
$$

More generally, if $\left|Q \cap \mathcal{H}_{\mathcal{B}, \gamma}^{j(N+2)}(E)\right| \geq \gamma$ we have $Q \subset \mathcal{H}_{\mathcal{B}, \gamma}^{j(N+2)+1}(E)$, or otherwise we may obtain

$$
\left|Q \cap \mathcal{H}_{\mathcal{B}, \gamma}^{(j+1)(N+2)}(E)\right| \geq\left(\frac{1}{\gamma}\right)^{j+1}\left|E_{0}\right|
$$

Now, let $\tilde{N}$ be a positive integer such that $\alpha \cdot\left(\frac{1}{\gamma}\right)^{\tilde{N}} \geq \gamma$. As $\left|E_{0}\right|=\alpha$ we have $\left(\frac{1}{\gamma}\right)^{\tilde{N}}\left|E_{0}\right| \geq \gamma$. Hence for some $m \leq(N+2) \cdot \tilde{N}$ we have $\left|Q \cap \mathcal{H}_{\mathcal{B}, \gamma}^{m}(E)\right| \geq \gamma$. In particular, $Q \subset \mathcal{H}_{\mathcal{B}, \gamma}^{(N+2) \tilde{N}+1}(E)$. As any integer greater than or equal to $\frac{\log ^{+}\left(\gamma \cdot 2^{n}\right)}{\log \left(\frac{1}{\gamma}\right)}$ 
would be acceptable for $N$ and any integer greater than or equal to $\frac{-\log \left(\frac{\gamma}{\alpha}\right)}{\log \gamma}$ would be acceptable for $\tilde{N}$, we obtain the lemma, where

$$
K_{\alpha, \gamma}=\left\lceil\frac{-\log \left(\frac{\gamma}{\alpha}\right)}{\log \gamma}\right\rceil \cdot\left\lceil 2+\frac{\log ^{+}\left(\gamma \cdot 2^{n}\right)}{\log \left(\frac{1}{\gamma}\right)}\right\rceil+1 .
$$

We now complete the proof of Proposition 1. As $\left|\left\{x: M_{\mathcal{B}} \chi_{E}(x)>\gamma\right\}\right| \leq C|E|$ for every measurable set $E$ if and only if $\left|\left\{x: M_{\mathcal{B}} \chi_{E}(x) \geq \gamma\right\}\right| \leq C|E|$ for every measurable set $E$, by the Tauberian condition on $M_{\mathcal{B}}$ we have that

$$
\left|\mathcal{H}_{\mathcal{B}, \gamma}^{k+1}(E)\right| \leq C_{\gamma}\left|\mathcal{H}_{\mathcal{B}, \gamma}^{k}(E)\right|
$$

holds for any positive integer $k$ and any measurable set $E$. An immediate consequence of the above lemma is that $\left\{x: M_{\mathcal{B}} \chi_{E}(x)>\alpha\right\} \subset \mathcal{H}_{\mathcal{B}, \gamma}^{K_{\alpha, \gamma}}(E)$, and hence

$$
\begin{aligned}
\left|\left\{x: M_{\mathcal{B}} \chi_{E}(x)>\alpha\right\}\right| & \leq\left|\mathcal{H}_{\mathcal{B}, \gamma}^{K_{\alpha, \gamma}}(E)\right| \\
& \leq C_{\gamma}\left|\mathcal{H}_{\mathcal{B}, \gamma}^{K_{\alpha, \gamma}-1}(E)\right| \\
& \leq \ldots \leq C_{\gamma}^{K_{\alpha, \gamma}}|E| .
\end{aligned}
$$

So $\left|\left\{x: M_{\mathcal{B} \chi_{E}}(x)>\alpha\right\}\right| \leq C_{\alpha, \gamma}|E|$, where $C_{\alpha, \gamma}=C_{\gamma}^{K_{\alpha, \gamma}}$ and $K_{\alpha, \gamma}$ is as in (1).

In Proposition $1 \mathcal{B}$ is a homothecy invariant collection of rectangular parallelepipeds. The following theorem is a generalization of Proposition 1 in that we allow $\mathcal{B}$ to be a homothecy invariant collection of convex sets.

Theorem 1. Let $\mathcal{B}$ be a homothecy invariant collection of convex sets in $\mathbb{R}^{n}$. Suppose for some $0<\alpha<1$ there exists $0<C_{\alpha}<\infty$ such that

$$
\left|\left\{x: M_{\mathcal{B}} \chi_{E}(x)>\alpha\right\}\right| \leq C_{\alpha}|E|
$$

holds for all measurable sets $E$ in $\mathbb{R}^{n}$. Then if $\delta>0$, there exists $0<C_{\alpha, \delta}<\infty$ such that

$$
\left|\left\{x: M_{\mathcal{B}} \chi_{E}(x)>\delta\right\}\right| \leq C_{\alpha, \delta}|E|
$$

holds for all measurable sets $E$ in $\mathbb{R}^{n}$, where $C_{\alpha, \delta}$ depends only on $C_{\alpha}, \alpha, \delta$, and the dimension $n$.

Proof. Given an ellipsoid $\mathcal{E}$ in $\mathbb{R}^{n}$ and $c>0$, we let $c \mathcal{E}$ denote the $c$-fold dilate of $\mathcal{E}$ that has the same center and orientation as $\mathcal{E}$.

Let $S \in \mathcal{B}$. As was proven by F. John in [4] (see also the related article [1] by K. Ball), since $S$ is convex there exists an ellipsoid $\mathcal{E}_{S}$ contained in $S$ such that $S \subset n \mathcal{E}_{S}$. Let $R_{S}$ be a rectangular parallelepiped containing $n \mathcal{E}_{S}$ of smallest possible volume. Note that $\left|R_{S}\right|<2^{n}\left|n \mathcal{E}_{S}\right|$ and hence $\left|R_{S}\right|<2^{n} \cdot n^{n}|S|$. Moreover, letting $c S$ denote the $c$-fold dilate of $S$ about the center of $\mathcal{E}_{S}$ we have $R_{S} \subset 2 n S$, since $R_{S} \subset 2 n \mathcal{E}_{S}$ and $2 n \mathcal{E}_{S} \subset 2 n S$.

Let $\tilde{\mathcal{B}}=\left\{R_{S}: S \in \mathcal{B}\right\}$. We may assume without loss of generality that the $\mathcal{E}_{S}$ and $R_{S}$ above are such that $\tilde{\mathcal{B}}$ is homothecy invariant.

Note that $M_{\tilde{\mathcal{B}}} f(x) \leq 2^{n} \cdot n^{n} M_{\mathcal{B}} f(x)$.

We now fix $\gamma$ such that $0<\alpha<\gamma<1$. 
Let $\rho=\frac{1}{2^{n} \cdot n^{n}}$. Also let

$$
\epsilon=\frac{\gamma-\alpha}{2-\gamma-\alpha} \rho \quad \text { and } \quad N=\left\lceil\frac{\log \left(1-\frac{2(1-\gamma)}{2-\gamma-\alpha}\right)}{\log \left(1-\rho-\frac{\gamma-\alpha}{2-\gamma-\alpha} \rho\right)}\right\rceil \text {. }
$$

One can show that

$$
\rho \frac{1-(1-\rho-\epsilon)^{N+1}}{\rho+\epsilon}>\frac{1-\gamma}{1-\alpha} .
$$

We will need the following technical lemma.

Lemma 2. Let $\epsilon>0$ be as above and $S$ be a convex set in $Q=[0,1]^{n}$.

Let $m \in \mathbb{N}$ be the unique positive integer such that

$$
\frac{\epsilon}{4 n} \leq \sqrt{n} 2^{-m}<\frac{\epsilon}{2 n} \text {. }
$$

Then there exists a set of cubes $\left\{Q_{j}\right\}$ of sidelength $2^{-m}$ such that

i) all the cubes $Q_{j}$ lie in $Q$ and are members of the mesh $\mathcal{M}_{m}$ of dyadic cubes of sidelength $2^{-m}$,

ii) each $Q_{i}$ is disjoint from $S$, and

iii) $\left|\cup Q_{i} \cup S\right| \geq 1-\epsilon$.

Proof. Let $\mathcal{C}$ be the set of cubes in the mesh $\mathcal{M}_{m}$ that lie in $Q$ and are disjoint from $S$. Suppose $x \in Q \backslash S$ and $d(x, S)>\frac{\epsilon}{2 n}$. Then as the diameter of any cube in $\mathcal{M}_{m}$ is less than $\frac{\epsilon}{2 n}$, we have $x \in Q_{j}$ for some $Q_{j}$ in $\mathcal{C}$. So

$$
\left\{x \in Q: d(x, S)>\frac{\epsilon}{2 n}\right\} \subset \bigcup_{Q_{j} \in \mathcal{C}} Q_{j} .
$$

Now, since $S$ is convex,

$$
\left|\left\{x \in Q: 0<d(x, S)<\frac{\epsilon}{2 n}\right\}\right|<2 n \cdot \frac{\epsilon}{2 n}=\epsilon,
$$

so the desired result holds.

If $S$ is a set in $\mathbb{R}^{n}$ and $\tau$ is a translation operator given by $\tau f(x)=f(x-\sigma)$ for some $\sigma \in \mathbb{R}^{n}$, we let $\tau S$ denote the set such that $\chi_{\tau S}(x)=\chi_{S}(x-\sigma)$. For each $c>0$ and set $S$ in $\mathbb{R}^{n}$ we define the set $\delta_{c} S$ to be such that $\chi_{\delta_{c} S}(x)=\chi_{S}\left(\frac{1}{c} x\right)$.

Lemma 3. Suppose $R \in \tilde{\mathcal{B}}$. Let $S \in \mathcal{B}$ such that $S \subset R,|R|<2^{n} \cdot n^{n}|S|$, and $R \subset 2 n S$. Then there exists an a.e. disjoint collection $\left\{S_{j}\right\}$ of translates of dilates of $S$ and a collection of translation operators $\left\{\tau_{j}\right\}$ such that $S_{j} \subset R$ for each $j$, $\left|\cup S_{j}\right|>\frac{1-\gamma}{1-\alpha}|R|$, and $R \subset \tau_{j} \delta_{2^{N m+n}} S_{j}$ for each $j$. (Here $m$ is as given by Lemma 2 and $N$ is as in (2).)

Proof. As the techniques of this proof are invariant under affine transformation, we may assume without loss of generality that $R=Q=[0,1]^{n}$.

Note that $\frac{|S|}{|R|}>\rho$.

By Lemma 2, there exists a collection $\left\{Q_{j}\right\}$ of (a.e.) disjoint $n$-cubes contained in $R$ and disjoint from $S$ lying in the mesh $\mathcal{M}_{m}$ such that $\left|\cup Q_{i} \cup S\right| \geq 1-\epsilon$.

Now let $\left\{\tau_{j}\right\}$ be a collection of translation operators such that $Q_{j}=\tau_{j} \delta_{2^{-m}} R$ for each $j$. 
Let $S_{1, j}=\tau_{j} \delta_{2^{-m}} S$. Note that

$$
\left|S \cup\left(\cup S_{1, j}\right)\right| \geq \rho+(1-\rho-\epsilon) \rho
$$

since $\left|\left(\cup Q_{j}\right) \cup S\right| \geq 1-\epsilon$ and $|S|>\rho$.

Let $S_{1}=S \cup\left(\cup S_{1, j}\right)$ and let $S_{2, j}=\tau_{j} \delta_{2^{-m}} S_{1}$. Observe that

$$
\left|S \cup\left(\cup S_{2, j}\right)\right| \geq \rho+(1-\rho-\epsilon) \rho+(1-\rho-\epsilon)^{2} \rho .
$$

Let $S_{2}=S \cup\left(\cup S_{2, j}\right)$.

We proceed by induction. $S_{k+1, j}$ and $S_{k+1}$ may be obtained from $S_{k}$ via

$$
S_{k+1, j}=\tau_{j} \delta_{2-m} S_{k}
$$

and

$$
S_{k+1}=S \cup\left(\cup S_{k+1, j}\right) .
$$

Note that

$$
\left|S \cup\left(\cup_{j} S_{k+1, j}\right)\right| \geq \rho+(1-\rho-\epsilon) \rho+\ldots+(1-\rho-\epsilon)^{k+1} \rho .
$$

Now recall $N$ is such that

$$
\rho \frac{1-(1-\rho-\epsilon)^{N+1}}{\rho+\epsilon}>\frac{1-\gamma}{1-\alpha} .
$$

So

$$
\begin{aligned}
\left|S_{N}\right| & \geq \rho+(1-\rho-\epsilon) \rho+\ldots+(1-\rho-\epsilon)^{N} \rho \\
& =\rho \frac{1-(1-\rho-\epsilon)^{N+1}}{1-(1-\rho-\epsilon)}=\rho \frac{1-(1-\rho-\epsilon)^{N+1}}{\rho+\epsilon} \\
& >\frac{1-\gamma}{1-\alpha} .
\end{aligned}
$$

Note also that there exists a collection of translation operators $\tau_{j, k}$ such that

$$
S_{N}=S \cup\left(\cup_{j=1}^{N} \cup_{k} \tau_{j, k} \delta_{2^{-j m}} S\right),
$$

where the union above is disjoint. So in particular $S_{N}$ may be expressed as the disjoint union $\cup S_{j}^{\prime}$, where $\left|\cup S_{j}^{\prime}\right|>\frac{1-\gamma}{1-\alpha}$ and each $S_{j}^{\prime}$ is a translate of a dilate of $S$ such that $S_{j}^{\prime} \subset R$. Moreover there exists a set of translation operators $\left\{\tau_{j}^{\prime}\right\}$ such that $S \subset \tau_{j}^{\prime} \delta_{2^{N m}} S_{j}^{\prime}$ for each $j$. Since $R \subset 2 n S$, there also exists a collection of translation operators $\left\{\tau_{j}^{\prime \prime}\right\}$ such that $R \subset \tau_{j}^{\prime \prime} \delta_{2^{N m+n}} S_{j}^{\prime}$ for each $j$. Relabeling $\left\{S_{j}^{\prime}\right\}$ as $\left\{S_{j}\right\}$ and $\left\{\tau_{j}^{\prime \prime}\right\}$ as $\left\{\tau_{j}\right\}$, we complete the proof of the lemma.

The following lemma shows that, since $M_{\mathcal{B}}$ satisfies a Tauberian condition with respect to $\alpha$, the maximal operator $M_{\tilde{\mathcal{B}}}$ satisfies a Tauberian condition with respect to any $\gamma$ greater than $\alpha$.

Lemma 4. If $\alpha<\gamma<1$, there exists $0<C_{\alpha, \gamma}^{\prime}<\infty$ such that

$$
\left|\left\{x: M_{\tilde{\mathcal{B}}} \chi_{E}(x)>\gamma\right\}\right| \leq C_{\alpha, \gamma}^{\prime}|E|
$$

holds for all measurable sets $E$ in $\mathbb{R}^{n}$, where $C_{\alpha, \gamma}^{\prime}$ depends only on $C_{\alpha}, \alpha, \gamma$, and the dimension $n$. 
Proof. Let $E$ be a measurable set in $\mathbb{R}^{n}$. Suppose $R \in \tilde{\mathcal{B}}$ and $\frac{1}{|R|} \int_{R} \chi_{E}>\gamma$. Let $\left\{S_{j}\right\}$ be as in Lemma 3. Then there exists $\tilde{S} \in\left\{S_{j}\right\}$ such that $\frac{1}{|\tilde{S}|} \int_{\tilde{S}} \chi_{E}>\alpha$, as otherwise

$$
\begin{aligned}
|E \cap R| & \leq\left(\frac{1-\gamma}{1-\alpha} \cdot \alpha+1 \cdot\left(1-\frac{1-\gamma}{1-\alpha}\right)\right)|R| \\
& =\gamma|R|,
\end{aligned}
$$

contradicting the fact that $|E \cap R| /|R|>\gamma$. By Lemma 3 we have $R \subset \tau \delta_{2^{N m+n}} \tilde{S}$ for some translation operator $\tau$. We now define $\Delta_{\alpha, \gamma}$ by

$$
\begin{aligned}
\Delta_{\alpha, \gamma}=1+\frac{n \log 2}{\log \left(\frac{1}{\alpha}\right)}( & {\left[\frac{\log \left(1-\frac{2(1-\gamma)}{2-\gamma-\alpha}\right)}{\log \left(1-\frac{1}{2^{n} n^{n}}-\frac{\gamma-\alpha}{2-\gamma-\alpha} \frac{1}{2^{n} n^{n}}\right)}\right] } \\
& {\left.\left[-\frac{\log \left(\frac{\gamma-\alpha}{2-\gamma-\alpha} \frac{1}{2^{n+1} n^{n+\frac{3}{2}}}\right)}{\log 2}\right\rceil+n\right) . }
\end{aligned}
$$

One can show that $\Delta_{\alpha, \gamma}$ satisfies the inequality

$$
\left(\frac{1}{\alpha}\right)^{\frac{1}{n}\left(\Delta_{\alpha, \gamma}-1\right)} \geq 2^{N m+n} .
$$

Note then that $R \subset \mathcal{H}_{\mathcal{B}, \alpha}^{\Delta_{\alpha, \gamma}-1}(\tilde{S})$ and in particular that $R \subset \mathcal{H}_{\mathcal{B}, \alpha}^{\Delta_{\alpha, \gamma}}(E)$. As $R$ is arbitrary in $\tilde{\mathcal{B}}$ subject to the condition that $\frac{1}{|R|} \int_{R} \chi_{E}>\gamma$, we then have

$$
\left\{x: M_{\tilde{\mathcal{B}}} \chi_{E}(x)>\gamma\right\} \subset \mathcal{H}_{\mathcal{B}, \alpha}^{\Delta_{\alpha, \gamma}}(E) .
$$

By the Tauberian condition on $M_{\mathcal{B}}$ we then have that

$$
\left|\left\{x: M_{\tilde{\mathcal{B}}} \chi_{E}(x)>\gamma\right\}\right| \leq C_{\alpha}^{\Delta_{\alpha, \gamma}}|E| .
$$

As $C_{\alpha}^{\Delta_{\alpha, \gamma}}$ depends only on $C_{\alpha}, \alpha, \gamma$, and $n$, and the desired result holds.

We now come to the end of the proof of the main theorem. We may assume $0<\delta<\alpha$ without loss of generality. The hypotheses of the theorem and Lemma 4 and its proof imply that $\left|\left\{x: M_{\tilde{\mathcal{B}}} \chi_{E}(x)>\gamma\right\}\right| \leq C_{\alpha}^{\Delta_{\alpha, \gamma}}|E|$ for $\alpha<\gamma<1$. We now set $\gamma=\tilde{\alpha}=\frac{1+\alpha}{2}$. Since $\tilde{\mathcal{B}}$ is a homothecy invariant collection of rectangular parallelepipeds, by the closing comments of the proof of Proposition 1 we have that for any measurable set $E$ in $\mathbb{R}^{n}$

$$
\left|\left\{x: M_{\tilde{\mathcal{B}}} \chi_{E}(x)>\frac{\delta}{2^{n} n^{n}}\right\}\right| \leq C_{\alpha}^{\Delta_{\alpha, \tilde{\alpha}} K \frac{\delta}{2^{n^{n} n}, \tilde{\alpha}}}|E| .
$$

Since $M_{\mathcal{B}} f(x) \leq 2^{n} n^{n} M_{\tilde{\mathcal{B}}} f(x)$ we then have

$$
\left|\left\{x: M_{\mathcal{B}} \chi_{E}(x)>\delta\right\}\right| \leq C_{\alpha}^{\Delta_{\alpha, \tilde{\alpha}} K_{\frac{\delta}{n^{n} n}, \tilde{\alpha}}}|E| .
$$

As $\Delta_{\alpha, \tilde{\alpha}}$ and $K_{\frac{\delta}{2^{n} n^{n}}, \tilde{\alpha}}$ depend only on $\alpha, \delta$, and $n$, the desired result holds.

We now show that the proof of the above result implies that, if $\mathcal{B}$ is a homothecy invariant collection of convex sets in $\mathbb{R}^{n}$ and the associated maximal operator $M_{\mathcal{B}}$ satisfies a Tauberian condition with respect to some $0<\alpha<1$, then $M_{\mathcal{B}}$ must be bounded on $L^{p}\left(\mathbb{R}^{n}\right)$ for sufficiently large $p$. 
Corollary 1. Let $\mathcal{B}$ be a homothecy invariant collection of convex sets in $\mathbb{R}^{n}$. Suppose for some $0<\alpha<1$ there exists a positive finite constant $C_{\alpha}$ such that

$$
\left|\left\{x: M_{\mathcal{B}} \chi_{E}(x)>\alpha\right\}\right| \leq C_{\alpha}|E|
$$

holds for every measurable set $E$ in $\mathbb{R}^{n}$. Then $M_{\mathcal{B}}$ is bounded on $L^{p}\left(\mathbb{R}^{n}\right)$ for sufficiently large $p$. In particular, there exists $p_{\alpha}<\infty$ depending only on $\alpha, n$, and $C_{\alpha}$ such that $M_{\mathcal{B}}$ is bounded on $L^{p}\left(\mathbb{R}^{n}\right)$ for all $p>p_{\alpha}$.

Proof. Let $\delta<\min \left(\frac{1}{100}, \alpha\right)$. By the closing remarks of the proof of Theorem 1 we have that

$$
\begin{aligned}
& \left|\left\{x: M_{\mathcal{B}} \chi_{E}(x)>\delta\right\}\right| \leq C_{\alpha}^{\Delta_{\alpha, \tilde{\alpha}} K \frac{\delta}{2^{n} n^{n}}, \tilde{\alpha}}|E| \\
& \leq C_{\alpha}^{\Delta_{\alpha, \tilde{\alpha}}}\left(\left[\frac{-\log \left(\frac{\tilde{\alpha} n^{n} n^{n}}{\tilde{\delta}}\right)}{\log \tilde{\alpha}}\right] \cdot\left[2+\frac{\log +\left(2^{n} \tilde{\alpha}\right)}{\log \left(\frac{1}{\tilde{\alpha}}\right)}\right]+1\right)|E| \\
& \leq C_{\alpha}^{\Delta_{\alpha, \tilde{\alpha}}} C_{\alpha}^{2 \Delta_{\alpha, \tilde{\alpha}} \frac{-\log \left(\frac{\left.\tilde{\alpha} 2^{n} n^{n}\right)}{\log \tilde{\alpha}}\right)}{\log } \cdot\left[2+\frac{\log +\left(2^{n} \tilde{\alpha}\right)}{\log \left(\frac{1}{\alpha}\right)} \mid\right.}|E| \\
& \leq C_{\alpha}^{\Delta_{\alpha, \tilde{\alpha}}}\left(\frac{\tilde{\alpha} \cdot 2^{n} \cdot n^{n}}{\delta}\right) \frac{\frac{-2 \log C_{\alpha}\left[2+\frac{\log +\left(2^{n} \tilde{\alpha}\right)}{\log \left(\frac{1}{\alpha}\right)}\right] \Delta_{\alpha, \tilde{\alpha}}}{\log \tilde{\alpha}}}{|E| . ~}
\end{aligned}
$$

Hence $M_{\mathcal{B}}$ is of restricted weak type $\left(p_{\alpha}, p_{\alpha}\right)$, where

$$
p_{\alpha}=\frac{-2 \log C_{\alpha}\left[2+\frac{\log ^{+}\left(2^{n} \tilde{\alpha}\right)}{\log \left(\frac{1}{\alpha}\right)}\right] \Delta_{\alpha, \tilde{\alpha}}}{\log \tilde{\alpha}},
$$

and hence $M_{\mathcal{B}}$ is bounded on $L^{p}\left(\mathbb{R}^{n}\right)$ for any $p>p_{\alpha}$. As $p_{\alpha}$ depends only on $\alpha, n$, and $C_{\alpha}$, the desired result follows.

Recall that a collection of sets in $\mathbb{R}^{n}$ is said to be a density basis if it differentiates $L^{\infty}\left(\mathbb{R}^{n}\right)$. We conclude this paper by observing the rather striking result that any density basis consisting of a homothecy invariant collection of convex sets in $\mathbb{R}^{n}$ must differentiate $L^{p}\left(\mathbb{R}^{n}\right)$ for sufficiently large $p$.

Corollary 2. Let $\mathcal{B}$ be a density basis that is a homothecy invariant collection of convex sets in $\mathbb{R}^{n}$. Then $\mathcal{B}$ differentiates $L^{p}\left(\mathbb{R}^{n}\right)$ for sufficiently large $p$.

Proof. Suppose $\mathcal{B}$ is a density basis that is a homothecy invariant collection of convex sets in $\mathbb{R}^{n}$. Then since $\mathcal{B}$ is a Busemann-Feller basis that is invariant by homothecies, we know for some $0<C<\infty$ that $\left|\left\{x: M_{\mathcal{B}} \chi_{E}(x)>\frac{1}{2}\right\}\right| \leq C|E|$ holds for all measurable sets $E$ in $\mathbb{R}^{n}$. (See p. 69 of $[3$ for a proof of this result.) By Corollary 1 we then have that $M_{\mathcal{B}}$ is bounded on $L^{p}\left(\mathbb{R}^{n}\right)$ for sufficiently large $p$ and hence $\mathcal{B}$ differentiates $L^{p}\left(\mathbb{R}^{n}\right)$ for sufficiently large $p$.

\section{REFERENCES}

[1] K. Ball, An elementary introduction to modern convex geometry. Flavors of geometry, 158, Math. Sci. Res. Inst. Publ., 31, Cambridge Univ. Press, Cambridge, 1997. MR1491097 (99f:52002)

[2] A. Córdoba and R. Fefferman, On the equivalence between the boundedness of certain classes of maximal and multiplier operators in Fourier analysis, Proc. Nat. Acad. Sci. USA 74 (1977), 423-425. MR0433117(55:6096)

[3] M. de Guzmán, Differentiation of Integrals in $R^{n}$, Lecture Notes in Math. 481, Springer, 1975. MR0457661 (56:15866) 
[4] F. John, Extremum problems with inequalities as subsidiary conditions. Studies and Essays Presented to R. Courant on his 60th Birthday, January 8, 1948, 187-204. Interscience Publishers, Inc., New York, N. Y., 1948. MR0030135 (10:719b)

[5] E. M. Stein, Singular Integrals and Differentiability Properties of Functions, Princeton University Press, 1970. MR0290095 (44:7280)

[6] E. M. Stein and G. Weiss, Introduction to Fourier Analysis on Euclidean Spaces. Princeton University Press, 1971. MR0304972 (46:4102)

[7] A. Zygmund, Trigonometric Series, Vol. 2, Cambridge Univ. Press, 1958.

Department of Mathematics, Baylor University, Waco, Texas 76798

E-mail address: paul_hagelstein@baylor.edu

Department of Mathematics, DePaul University, Chicago, Illinois 60614

E-mail address: astokolo@math.depaul.edu 